\title{
PROPAGATION OF HIGHLY NONLINEAR SIGNALS IN A TWO DIMENSIONAL NETWORK OF GRANULAR CHAINS
}

\author{
C. Daraio ${ }^{1}$, V. F. Nesterenko ${ }^{2}$ \\ ${ }^{1}$ Aeronautics (GALCIT) and Applied Physics, California Institute of Technology, Pasadena, CA 91125, 105-50, \\ ${ }^{2}$ Mechanical and Aerospace Engineering Department, University of California at San Diego, La Jolla, CA \\ 92093-0411
}

\begin{abstract}
We report the first experimental observation of highly nonlinear signals propagating in a two dimensional system composed of granular chains. In this system one of the chains contacts two others to allow splitting and redirecting the solitary-like signal formed in the first chain. The system consists of a double Y-shaped guide in which high- and low-modulus chains of spheres are arranged in various geometries. We observed fast splitting of the initial pulse, rapid chaotization of the signal and sharp bending of the propagating acoustic information. Pulse and energy trapping was also observed in composite systems assembled from hard- and soft- particles in the branches.
\end{abstract}

Keywords: Granular materials, nonlinear waves, solitary waves, dynamic compression

PACS: 05.45.Yv, 46.40.Cd, 43.25.+y, 45.70.-n

\section{INTRODUCTION}

Granular chains represent one of the most studied examples of highly nonlinear systems exhibiting a very unique wave behavior in one dimension [1-3]. Recently, the study of the highly nonlinear behavior at the interface between two highly nonlinear media has received increasing attention due to the discovery of interesting new dynamical aspects arising from the selforganization of the granules in close proximity to the interface [4-7]. The three dimensional structural features of the granular state include filamentary force (stress) chains, i.e. chains of granules with preferred contacts for force/pulse transmission. Dynamic stress chains can be a source of hot spot formation and was studied in [8] for two dimensional particle beds.

The passage of a highly nonlinear solitary wave from a one dimensional chain of beads through a two dimensional geometrical bifurcation is also a matter of interest due to non uniqueness of the outcome of this interaction based on conservation laws of energy and momentum and due to possible application in acoustic signals transmission and transformation.

In this manuscript we describe the experimental observation of highly nonlinear solitary waves propagating through such interfaces. We study uniform systems assembled from PTFE (polytetrafluoroethylene) and stainless steel beads, as well as heterogeneous structures composed by a combination of steel and polymer spheres including Parylene-C coated steel beads.

\section{EXPERIMENTAL SET-UP AND RESULTS}

To create the two dimensional systems, we used a total number of 132 beads of uniform diameter, $(4.76 \mathrm{~mm})$ composed of different materials: the high mass, high elastic modulus steel (non-magnetic, 316 type) beads [4,5,9]; the small mass, low-modulus PTFE beads [6,9] and the high mass, low elastic modulus Parylene-C coated steel 
beads with slightly larger diameter $(4.86 \mathrm{~mm})$ [10]. The properties of the materials composing the beads used in this study are summarized in Table 1.

Table 1. Properties of the materials composing the highly nonlinear medium tested experimentally $[4,6,10]$.

\begin{tabular}{ccccc} 
Beads type & $\begin{array}{c}\text { Elastic } \\
\text { Modulus } \\
{[\mathrm{GPa}]}\end{array}$ & $\begin{array}{c}\text { Poisson } \\
\text { Ratio }\end{array}$ & $\begin{array}{c}\text { Density } \\
{\left[\mathrm{Kg} / \mathrm{m}^{3}\right]}\end{array}$ & $\begin{array}{c}\text { Bead } \\
\text { Mass } \\
{[\mathrm{g}]}\end{array}$ \\
\hline 316 Steel & 193 & 0.30 & 8000 & 0.45 \\
PTFE & 1.46 & 0.46 & 2200 & 0.123 \\
Parylene-C & 15 & 0.38 & 1289 & 0.45
\end{tabular}

The beads were arranged in a horizontal PTFE guide with $5 \mathrm{~mm}$ diameter grooves carved in a diamond-like, double- $Y$ shape with bifurcation angles of $60^{\circ}$ between the two branches (see Fig. 1(a) for a schematic). Four calibrated piezo-sensors were embedded inside particles in the system as described in $[5,6]$ and connected to a Tektronix oscilloscope, allowing the calculation of the signal's speed and the monitoring of the wave's shape. Pulses were generated with spherical steel strikers of different masses $(0.45 \mathrm{~g}$ and $5.33 \mathrm{~g})$ dropped at various impact velocities $(0.1-1.0 \mathrm{~m} / \mathrm{s})$.
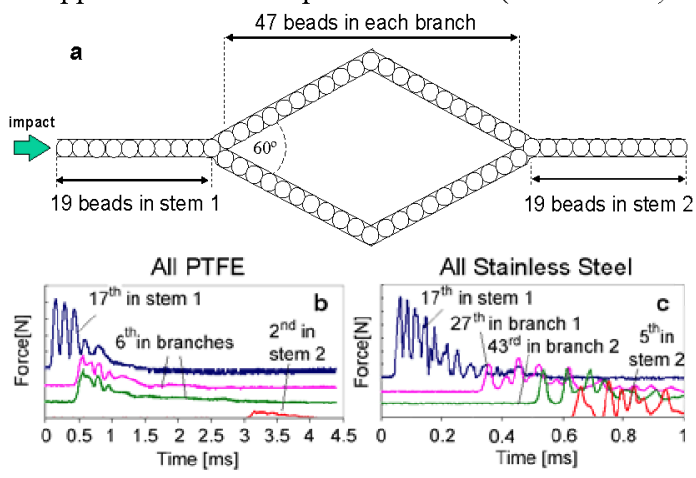

Figure 1. (Color Online) Trains of highly nonlinear waves propagating in uniform systems. (a) Experimental setup. (b) Waves excited by a $0.45 \mathrm{~g}$ striker in a PTFE based system. The y-axes scale for all curves is $0.2 \mathrm{~N}$ per division. (c) Waves excited by a $5.33 \mathrm{~g}$ striker in a stainless steel based system. The y-axes scale for the top curve is $2 \mathrm{~N}$ per division and $1 \mathrm{~N}$ for the other curves.

The first set of experiments was performed on uniform systems composed of all PTFE (Fig. 1(b)) or all stainless steel beads (Fig. 1(c)). Sensors were positioned in each one of the branches as pointed by the arrows in the corresponding curves, to monitor the shape and duration of the solitary waves before and after the bifurcated interface.

A second set of experiments was performed on composite systems with an asymmetric interface where the stainless steel beads in one of the branches were replaced with polymer based (low modulus and/or low mass) particles (see Fig. 2(a) for the stainless steel/PTFE based assembly).

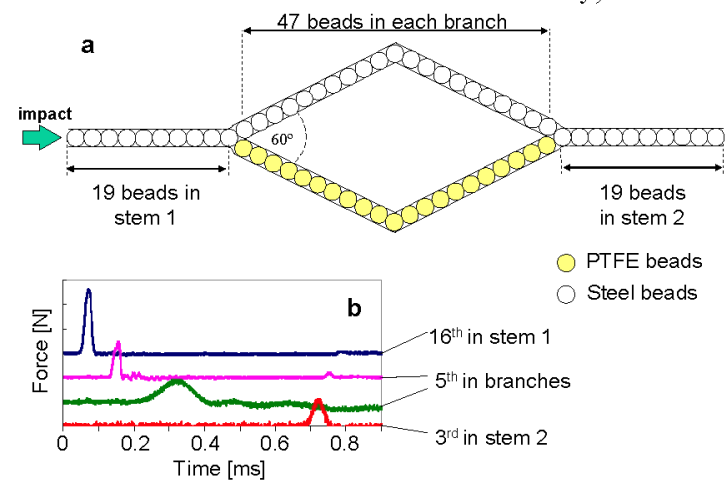

Figure 2. (Color Online) Highly nonlinear waves propagating in the diamond-shaped holder filled with stainless steel and PTFE beads. (a) Schematic diagram of the geometrical arrangement used. (b) Experimental data corresponding to the train of solitary waves excited by a $0.45 \mathrm{~g}$ striker. The $\mathrm{y}$-axes scale for the top two curves is 2 $\mathrm{N}$ per division, while it is $0.2 \mathrm{~N}$ for the two lower curves.

This set up was chosen to analyze the wave behavior in highly nonlinear systems having a completely new type of interfaces, connecting systems with different masses and signal's speeds. This arrangement is important to understand the fundamental mechanisms governing the responses of force chains in three dimensional systems and also to create practical devices for signal phaseshifting, pulse scrambling and energy trapping within one of the diamond branches. Results obtained in the stainless steel/PTFE based system are presented in Fig 2(b), where a $0.45 \mathrm{~g}$ striker was used to excite a single solitary wave in the first 19 beads before the interface.

The last system tested was arranged similarly to the previous case (Fig. 2(a)), with Parylene-C coated steel beads replacing the PTFE particles. In this case the mass of the particles was maintained uniform, and only the value of the elastic modulus was varied between the two branches. The 
experimental results for a single solitary wave and a train of solitary waves initially excited in the system are shown in Fig. 3(a) and (b) respectively.
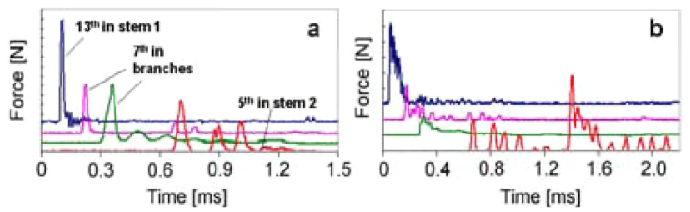

Figure 3. (Color Online) Highly nonlinear waves propagating in the stainless steel/Parylene-C beads system. (a) Experimental data excited by a $0.45 \mathrm{~g}$ striker. The y-axes scale is $2 \mathrm{~N}$ for the two top most curves, 0.4 $\mathrm{N}$ per division for the third curve and $0.1 \mathrm{~N}$ for the bottom curve. (b) Experimental data corresponding to the train of solitary waves excited by a $5.33 \mathrm{~g}$ striker, with sensors located in the same position as in (a). The $y$-axes scale for the three topmost curves is $5 \mathrm{~N}$ per division and $0.5 \mathrm{~N}$ for the last (bottom) curve.

\section{DISCUSSION}

The behavior of a train of solitary waves in a uniform system interacting with a symmetrically bifurcated interface and later merging back into a single chain is presented in Fig. 1(b,c) for PTFE and stainless steel beads correspondingly. In this particular system, the behavior of a single solitary wave crossing the interface may be predicted using conservation laws of momentum and energy for the solitons before and after the interface using the concept of effective mass of a solitary wave equal to about 1.4 times the mass of one particle in the chain [11]. The amplitude and speed of the solitary wave traveling in each branch after the bifurcation is expected to be slightly larger than the half of their values in the incoming pulse. This case is analogous to the well known behavior of a billiard ball hitting with velocity $v_{\mathrm{p}}$ the center of two other balls of equal mass and properties at rest. Although, there is a qualitative difference between the impact of 3 balls and the interaction of a highly nonlinear solitary wave with a Y-shaped junction between uniform chains. It is due to the fact that energy and momentum conservation laws result in uniquely defined velocities of the billiard balls after their interaction, while there are an infinite numbers of combinations of transmitted and reflected solitary waves which can satisfy conservation laws in the latter case.
We tailor the behavior of a train of solitary waves formed in the first stem, splitting system into two branches at the Y-interface, and later recombining together. For the PTFE based system (Fig. 1(b)), the wave train, after crossing the first interface, appears to split in two identical trains, with amplitude about half of its initial one before crossing the bifurcation. From a purely theoretical prospective, the expected reduction of this pulse speed has been calculated using Eq. 3 in [9] from the initial $129.8 \mathrm{~m} / \mathrm{s}$ in the stem to $114.5 \mathrm{~m} / \mathrm{s}$ in the two branches. These theoretical values agree well with the experimental observations, obtained from time-of-flight velocity measurements, despite the significant presence of dissipation. The absence of any evident reflected pulse in the branches, and the ability of the waves in the branches to recombine together in the final stem, demonstrates the capability of the pulses in such system to undergo $150^{\circ}$ and $120^{\circ}$ bends without major losses. Similar results were observed also for the stainless steel based system. It is important to mention that this specific design may result in undesirable reflections caused by the interactions between the pulse and the walls of the guides around the bends.

Next, we tailored the response of the system using non-uniform branches. The first of such systems featured the presence of an asymmetric stainless steel/PTFE interface (Fig. 2(a)). This particular geometry was selected because the two materials have a well known and extensively studied highly nonlinear response $[4,6,10]$ and a large difference in the signal speed.

The single solitary wave in the initial chain was excited using a stainless steel bead as the striker. It is evident (Fig. 2(b)) that once the excited pulse reaches the interface it splits asymmetrically in the two branches. In the stainless steel side (second curve from the top in Fig. 2(b)) the systems still supports the propagation of a single solitary wave. In the PTFE side (see third curve from the top in Fig. 2(b)) the single pulse splits into a train of $\sim 4$ waves of similar duration in which the first (leading) pulse carries much higher force amplitude. In addition, such train of solitary waves formed in the softer PTFE section appears to remain trapped, in the time interval studied, between the two stems and to "bounce" back and forth with a behavior similar to [6], until it disappears due to the effects of dissipation. This 
phenomenon can be useful for the practical applications. It is interesting to notice that the solitary wave in the steel section appears to propagate undisturbed until the $4^{\text {th }}$ sensor in the second stem, undergoing one $120^{\circ}$ and one $150^{\circ}$ turn. The speed of the signal in the stainless steel branch is estimated to be $\sim 4$ times larger than the speed of pulse traveling in the softer PTFE branch.

Results obtained from an interface composed of stainless steel and Parylene-C coated beads are reported in Fig. 3. Such system creates a unique interface featuring particles with uniform mass in the two branches beyond the splitting but with different elastic moduli. Interestingly such set up behaved similarly to the previous steel/PTFE case. When a single incident solitary wave, excited in the initial chain, propagated through the first bifurcated interface, the signal split into two parts: one composed of a single solitary-like pulse in the bare stainless steel branch (see second curve from the top in Fig. 3(a)); and the other one composed of a train of 4-5 solitary-like pulses that propagated in the Parylene-C coated section (third curve in Fig. 3(a)). Such wave-train formation in the softer, but equally heavy, section was fairly unexpected but it could be explained by some complex dynamic response of the elastically softer polymeric coating. Pulse trapping analogue to the one reported for the PTFE case is also observed. These results are relevant to underline the presence of self phaseshifting in the system and they suggest possible applications of such structures as delay lines, pulse scramblers and chaotization devices.

Analogous results were obtained when testing the same beads configuration exciting a train of solitary pulses in the first stem of the system (see Fig. 3(b)). In this case, the $4^{\text {th }}$ curve shows clearly the separate arrival of the train of waves from the steel branch (at $\sim 0.6 \mathrm{~ms}$ from the triggering time) and from the Parylene coated section, at $\sim 1.4 \mathrm{~ms}$, triggering a "delay" effect on the pulse arrival in the final chain section. Besides the observation of the described incident and transmitted pulses, a complex dynamic "rattling" among the beads and reflections from the bends and the interfaces are also observed (see for example the secondary pulses in the $2^{\text {nd }}$ and $4^{\text {th }}$ curve of Fig. 3(a)). To provide a comprehensive explanation of the dynamic of this 2-D systems numerical simulations similar to $[4,6,10]$ are being developed.

\section{CONCLUSIONS}

In this work we studied the behavior of strongly nonlinear waves in a two-dimensional granular medium analogous to the typical force-chains present in three-dimensional assemblies. We describe the behavior of incident and transmitted pulses across interfaces and demonstrated experimentally the ability to bend, split, scramble, trap and delay single and trains of soliton-like pulses. The presented results provide a simple model system for tailoring the behavior of pulses propagating in multidimensional granular materials. In addition, we analyzed the behavior of a pulse propagating through the interface between steel and polymer coated steel beads and showed the unexpected decomposition of a single solitary wave into a train of $\sim 4-5$ separated pulses. The principles demonstrated here can be utilized for the design of new composite structures and devices.

\section{ACKNOWLEDGEMENTS}

This work was supported by the National Science Foundation (Grant No. DCMS03013220).

\section{REFERENCES}

1. Nesterenko, V.F. Prikl. Mekh. Tekh. Fiz. 24, 136 1983 [J. Appl. Mech. Tech. Phys. 24, 733, 1984].

2. Nesterenko, V. F.; Dynamics of Heterogeneous Materials, Chapter 1 (Springer-Verlag, NY, 2001).

3. Coste, C. \& Gilles, B. European Physical Journal B 7, 155, 1999.

4. Hong, J., Phys. Rev. Lett. 94, 108001, 2005.

5. Nesterenko, V. F., Daraio, C., Herbold, E. B., Jin, S. Phys. Rev. Lett., 95, 158702, 2005.

6. Daraio, C.; Nesterenko, V.F.; Herbold, E.; Jin, S. Phys. Rev. Lett., 96, 058002, 2006.

7. Job, S., Melo, F., Sen, S. \& Sokolow, A. Phys. Rev. Lett., 94, 178002, 2005.

8. Roessig, K.M., Foster, J.C., Jr. and Bardenhagen, S.G., Exp. Mech., 42, 329, 2002.

9. Daraio, C., Nesterenko, V. F., Herbold, E. B., Jin, S. Phys. Rev. E, 73, 026610, 2006.

10. Daraio, C., Nesterenko, V. F Phys. Rev E, 73, 26612, 2006.

11. Daraio, C.; Nesterenko, V.F.; Jin, S. APS - SCCM2003, 197-200, AIP Conference Proceedings, Editors M. D. Furnish, Y. M. Gupta, and J. W. Forbes, Portland (Oregon), 2004. 